CERN-TH.6569/92

CTP-TAMU-53/92

ACT $-15 / 92$

\title{
The prospects for CHORUS and NOMAD in the light of COBE and GALLEX
}

\author{
JOHN ELLIS ${ }^{(a)}$, JORGE L. LOPEZ ${ }^{(b)(c)}$, and D. V. NANOPOULOS ${ }^{(a)(b)(c)}$ \\ (a) CERN Theory Division, 1211 Geneva 23, Switzerland \\ (b) Center for Theoretical Physics, Department of Physics, Texas A\&M University \\ College Station, TX 77843-4242, USA \\ (c) Astroparticle Physics Group, Houston Advanced Research Center (HARC) \\ The Woodlands, TX rry381, USA
}

\begin{abstract}
The most natural MSW neutrino oscillation interpretation of the GALLEX and other solar neutrino data, which invokes $m_{\nu_{\mu}} \sim 3 \times 10^{-3} \mathrm{eV}$, and a general GUT see-saw hierarchy of neutrino masses, $m_{\nu_{e, \mu, \tau}} \sim\left(m_{u, c, t}\right)^{2} / M_{U}$, suggest that $m_{\nu_{\tau}} \sim 10 \mathrm{eV}$ in agreement with the preference of $\mathrm{COBE}$ and other data on large-scale structure in the Universe for a hot component in the Dark Matter. The general see-saw model also suggests that neutrino mixing angles are related to quark mixing angles, which is also consistent with the oscillation interpretation of the solar neutrino data, and suggests that the forthcoming CHORUS and NOMAD experiments at CERN have a good chance of observing $\nu_{\mu}-\nu_{\tau}$ oscillations. We present a minimal realization of the general see-saw hierarchy in the context of flipped $S U(5)$.
\end{abstract}

CERN-TH.6569/92

CTP-TAMU-53/92

ACT 15/92

June 1992 


\section{Introduction}

The recent GALLEX data [1] have added a fascinating new twist to the continuing saga of solar neutrinos. They find a solar neutrino deficit, as did the Homestake [2] and Kamioka [3] experiments, although seemingly not as large a deficit as that reported by the SAGE experiment [4]. The interpretation of these measurements is not yet clear, with explanations being sought in nuclear physics - are all the reaction rates correct? - in astrophysics - is the standard solar model correct? - and in particle physics - do neutrinos oscillate? We discard the nuclear hypothesis: it seems that the residual rate uncertainties are no longer sufficient to make the deficit go away [5]. More questionably, we also discard the astrophysical hypothesis: simply reducing the core temperature of the Sun would suppress the higher-energy Kamioka data more than the Homestake data, whereas the opposite seems to be the case, and helioseismological observations are by now severely constraining alternative solar models [6], [7].

We are left with the neutrino oscillation hypothesis, or rather hypotheses, since there are several possible oscillation scenaria [8]. These include vacuum oscillations with $\Delta m_{\nu}^{2} \sim$ $10^{-10} \mathrm{eV}^{2}$ and a large mixing angle $\theta$, as well as two matter-enhanced Mikheyev-SmirnovWolfenstein (MSW) [9] possibilities with $\Delta m_{\nu}^{2} \sim 10^{-5} \mathrm{eV}^{2}$ and $\sin ^{2} 2 \theta>1 / 2$ or $\sim 10^{-2}$. As discussed later, we find large mixing angles theoretically implausible, and therefore focus here on the $\Delta m_{\nu}^{2} \sim 10^{-5} \mathrm{eV}^{2}$ and $\sin ^{2} 2 \theta \sim 10^{-2}$ scenario. Again as reviewed later, a general GUT see-saw hierarchy mechanism $[10,11]$ suggests that $m_{\nu_{e, \mu, \tau}} \sim\left(m_{u, c, t}\right)^{2} / M_{U}$ so that $m_{\nu_{e}} \ll m_{\nu_{\mu}} \ll m_{\nu_{\tau}}$, and also that $\theta_{e \mu} \gg \theta_{e \tau}$, so we assume that the Sun is telling us about $\nu_{e}-\nu_{\mu}$ oscillations. ${ }^{1}$ The see-saw mechanism therefore suggests that $m_{\nu_{\mu}} \sim 3 \times 10^{-3} \mathrm{eV}$ and hence that $m_{\nu_{\tau}} \sim 10 \mathrm{eV}$.

We are impressed by the concordance between this numerology and the best-fit interpretation [13] of COBE [14] and other data on large-scale structure in the Universe, which includes a hot dark matter (HDM) component as well as the dominant cold dark matter (CDM) component. ${ }^{2}$ Although this may involve taking the data too seriously, it does seem as if the COBE data are most easily reconciled with the data on peculiar velocities and the galaxy-galaxy correlation function at large angles if $\Omega_{H D M} \sim 0.3$ and $\Omega_{C D M} \sim 0.7$ with

1 We discard the reports of $\nu_{e}-\nu_{\mu}$ oscillations in the cosmic-ray neutrino flux, which the type of model discussed here cannot reconcile with any of these solar neutrino oscillation scenaria: see however [12].

2 Such a scenario was suggested by K. A. Olive and one of us (D.V.N.) many years ago [15]. 
$\Omega_{B}<0.1$ [13]. If the $\nu_{\tau}$ constitutes this HDM, it should weigh several $\mathrm{eV}$, in agreement with the above estimate based on solar neutrino oscillations and the see-saw mechanism.

The purpose of this note is to link these remarks with the prospects for observing $\nu_{\mu}-\nu_{\tau}$ oscillations in the CHORUS [16] and NOMAD [17] experiments now being prepared at CERN. The above "consensus" value of $m_{\nu_{\tau}}$ is certainly big enough for the CERN experiments to detect $\nu_{\mu}-\nu_{\tau}$ oscillations if the $\nu_{\mu}-\nu_{\tau}$ mixing angle $\theta_{\mu \tau}$ is large enough. In fact, the general GUT see-saw mechanism links the neutrino mixing angles with the corresponding neutrino masses, at least qualitatively. We show that this theoretical expectation is consistent with the value of $\theta_{e \mu}$ indicated by the GALLEX and other solar neutrino experiments, and use it to estimate the magnitude of $\theta_{\mu \tau}$, finding that it does indeed put $\nu_{\mu}-\nu_{\tau}$ oscillations within reach of the CHORUS and NOMAD experiments, as well as the P803 proposal [18] at FNAL. We illustrate these remarks with a minimal realization of the general GUT see-saw mechanism in the framework of a generic field-theoretical flipped $\mathrm{SU}(5)$ model [19], showing explicitly how the phenomenologically interesting mass ratios arise in this model.

\section{Review of the GUT see-saw mechanism}

Small neutrino masses are most naturally realized in terms of the GUT see-saw mechanism [11,10], wherein the light left-handed neutrino fields $(\nu)$ of the Standard Model interact with new superheavy right-handed (Majorana) neutrino fields $\left(\nu^{c}\right)$ through Dirac mass terms, as follows

$$
\begin{aligned}
& \nu \quad \nu^{c} \\
& \nu^{c}\left(\begin{array}{cc}
0 & m \\
m & M
\end{array}\right) \longrightarrow m_{\nu} \approx \frac{m^{2}}{M}, \quad m_{\nu^{c}} \approx M .
\end{aligned}
$$

Each of the entries $(m, M)$ in the above matrix should actually be regarded as a $3 \times 3$ submatrix in generation space. The generic large mass-scale $M$ is normally related to $M_{U} \approx 10^{15}$ to $10^{19} \mathrm{GeV}$, and the " 0 " entry may also be $\mathcal{O}\left(\mathrm{m}^{2} / M\right)$ in some models. The Dirac mass term above appears quite naturally in GUTs, when $\nu^{c}$ is embedded in a suitable representation, such as a $\mathbf{1}$ of $S U(5)$ or a $\mathbf{1 6}$ of $S O(10)$ [20]. The difficulties and ambiguities arise when trying also to obtain a Majorana mass term for $\nu^{c}$. This term does not arise in a minimal $S U(5)$ GUT. In $S O(10)$ GUTs it can arise with the introduction of a $\mathbf{1 2 6}$ representation [11] with suitable vacuum expectation values and additional singlet fields 
[20]. The Dirac mass term in this case is generally given by the up-quark mass matrix. It should be stressed though that the details of this mechanism in $S O(10)$ GUTs tend to be rather complicated. For the time being we will simply assume that the neutrino masses indeed scale with the up-quark squared masses, ${ }^{3}$ that is, they obey the following mass hierarchy

$$
m_{\nu_{e}}: m_{\nu_{\mu}}: m_{\nu_{\tau}} \sim m_{u}^{2}: m_{c}^{2}: m_{t}^{2}
$$

with some correction factors to be discussed later. In principle, the see-saw mechanism would also predict the low-energy neutrino mixings in analogy with the CabibboKobayashi-Maskawa (CKM) quark mixings. However, this is a much more involved proposition, since the inter-generation dependence of the $6 \times 6$ see-saw matrix needs to be known.

Here we simply assume that the heavy neutrino degrees of freedom are integrated out and at low energies one ends up with an effective $3 \times 3$ neutrino mass matrix $M_{\nu}$. Without loss of generality, we can choose a basis where the charged lepton mass matrix is diagonal and therefore the orthogonal matrix (assuming that $M_{\nu}$ is symmetric) $V_{\nu}$, which diagonalizes $M_{\nu}$, contains the relevant mixing angles. Since we expect these angles to be small, we can parametrize this matrix as follows

$$
V_{\nu} \approx\left(\begin{array}{ccc}
1 & \theta_{e \mu} & 0 \\
\theta_{e \mu} & 1 & \theta_{\mu \tau} \\
0 & \theta_{\mu \tau} & 1
\end{array}\right)
$$

where we assume that the corresponding $\theta_{e \tau}$ angle is very small (see below).

\section{Predictions for CHORUS and NOMAD}

The neutrino mass ratios in Eq. (2.2) neglect the running of the parameters between the unification scale $M_{U}$ and the low-energy quark mass scale $\left(m_{q}\right.$ or $1 \mathrm{GeV}$, whichever is larger: below this scale the quark masses do not run any more). The ratios in Eq. (2.2) also assume a generation-independent Majorana mass $M$, which is not necessarily true in models, as we discuss later. Incorporating the running of the parameters and relaxing the assumption of generation-independence one obtains [21]

$$
\frac{m_{\nu_{e}}}{m_{\nu_{\mu}}}=\left[\frac{\lambda_{u}\left(M_{U}\right)}{\lambda_{c}\left(M_{U}\right)}\right]^{2} \frac{M_{2}}{M_{1}} \approx\left[\frac{m_{u}}{m_{c}}\right]^{2} \frac{M_{2}}{M_{1}},
$$

3 In Sec. 4 we present a simple realization of this statement based on the flipped $S U(5)$ gauge group [19]. 
where $m_{u} \equiv m_{u}(1 \mathrm{GeV})=5.1 \pm 1.5 \mathrm{MeV}$ and $m_{c} \equiv m_{c}\left(m_{c}\right)=1.27 \pm 0.05 \mathrm{GeV}$, use has been made of the common running of the up- and charm-quark Yukawa couplings, and we assume $M \equiv \operatorname{diag}\left(M_{1}, M_{2}, M_{3}\right)$. Also,

$$
\frac{m_{\nu_{\mu}}}{m_{\nu_{\tau}}}=\left[\frac{\lambda_{c}\left(M_{U}\right)}{\lambda_{t}\left(M_{U}\right)}\right]^{2} \frac{M_{3}}{M_{2}}=\left[\frac{\lambda_{c}\left(m_{t}\right)}{\lambda_{t}\left(m_{t}\right)}\right]^{2}\left[1-\frac{\lambda_{t}^{2}\left(m_{t}\right)}{\lambda_{C}^{2}}\right] \frac{M_{3}}{M_{2}}=\left[\frac{m_{c}}{\eta_{c} m_{t}}\right]^{2}\left[1-\left(\frac{m_{t}}{190}\right)^{2}\right] \frac{M_{3}}{M_{2}},
$$

where $\eta_{c} \equiv m_{c}\left(m_{c}\right) / m_{c}\left(m_{t}\right) \approx 1.9$ for a plausible range of values of $\alpha_{3}\left(M_{Z}\right)[21,22,23]$, and $\lambda_{C}$ is the critical value of the top-quark Yukawa coupling above which $\lambda_{t}$ develops a Landau pole below $M_{U}$; one obtains $\lambda_{t}\left(m_{t}\right) / \lambda_{C} \approx m_{t} / 190 \mathrm{GeV}$. The value of $\lambda_{C}$ is decreased by $\lesssim 5 \%$ if the bottom-quark Yukawa coupling is not neglected. In Eq. (3.2) we have used the following approximate formulae [23,24]

$$
\begin{aligned}
\lambda_{c}^{2}\left(m_{t}\right) & =\eta \lambda_{c}^{2}\left(M_{U}\right), \\
\lambda_{t}^{2}\left(m_{t}\right) & =\eta\left[1-\frac{\lambda_{t}^{2}\left(m_{t}\right)}{\lambda_{C}^{2}}\right] \lambda_{t}^{2}\left(M_{U}\right),
\end{aligned}
$$

where $\eta \equiv \prod_{i=1}^{3}\left(\alpha_{U} / \alpha_{i}\right)^{c_{i} / b_{i}}$ with $c_{i}=\left(\frac{13}{15}, 3, \frac{16}{3}\right)$ and $b_{i}=\left(\frac{33}{5}, 1,-3\right)$. Note the significant modification of the $m_{\nu_{\mu}} / m_{\nu_{\tau}}$ ratio due to the running of parameters involved.

For the neutrino mixing angles we make the following phenomenologically-motivated ansätze

$$
\theta_{e \mu}=\left(m_{\nu_{e}} / m_{\nu_{\mu}}\right)^{1 / 4} \quad \text { and } \quad \theta_{\mu \tau}=\left(m_{\nu_{\mu}} / m_{\nu_{\tau}}\right)^{1 / 2}
$$

which resemble predictions for the CKM angles based on certain textures for the quark mass matrices [25],[20]. Indeed, from Eqs. (3.1) and (3.2) one can verify that $\theta_{\mu \tau} \sim \theta_{e \mu}^{2}$ for a plausible range of the parameters, whilst $\theta_{e \tau}$ would be much smaller. Therefore, $V_{\nu}$ in Eq. (2.3) has the same texture as the CKM matrix, although with $\theta_{e \mu} \ll \theta_{c}$. Accounting for all the proper factors, the relations (3.4) give

$$
\sin ^{2} 2 \theta_{e \mu}=4\left[\frac{m_{u}}{m_{c}}\right]\left(\frac{M_{2}}{M_{1}}\right)^{1 / 2}
$$

and

$$
\sin ^{2} 2 \theta_{\mu \tau}=4\left[\frac{m_{c}}{\eta_{c} m_{t}}\right]^{2}\left[1-\left(\frac{m_{t}}{190}\right)^{2}\right] \frac{M_{3}}{M_{2}}
$$

which we now confront with experiment.

The prediction for $\sin ^{2} 2 \theta_{e \mu}$ in Eq. (3.5) gives a central value of $1.6 \times 10^{-2}\left(M_{2} / M_{1}\right)^{1 / 2}$. Since the best fits to the GALLEX data in terms of the MSW mechanism give $\Delta m_{\nu_{e}-\nu_{\mu}}^{2}=$ 
Table I: Values of the $\nu_{\mu}-\nu_{\tau}$ mixing parameters, $\tau$-neutrino mass, and relic cosmological density as a function of the top-quark mass [27] for the model discussed in Sec. 3 and central values of the parameters (including $M_{3} / M_{2}=10$ ). Where relevant we have assumed that $m_{\nu_{\mu}}=2 \times 10^{-3} \mathrm{eV}$.

\begin{tabular}{|c|c|c|c|c|}
\hline$m_{t}(\mathrm{GeV})$ & $\sin ^{2} 2 \theta_{\mu \tau}$ & $\Delta m_{\nu_{\mu}-\nu_{\tau}}^{2}\left(\mathrm{eV}^{2}\right)$ & $m_{\nu_{\tau}}(\mathrm{eV})$ & $\Omega_{\nu} h^{2}$ \\
\hline 90 & $1.7 \times 10^{-3}$ & 22 & 4.7 & 0.05 \\
100 & $1.3 \times 10^{-3}$ & 38 & 6.2 & 0.07 \\
110 & $9.8 \times 10^{-4}$ & 66 & 8.1 & 0.09 \\
120 & $7.5 \times 10^{-4}$ & 115 & 11 & 0.12 \\
130 & $5.6 \times 10^{-4}$ & 202 & 14 & 0.16 \\
140 & $4.2 \times 10^{-4}$ & 368 & 19 & 0.21 \\
150 & $3.0 \times 10^{-4}$ & 715 & 27 & 0.29 \\
\hline
\end{tabular}

$(0.3-1.2) \times 10^{-5} \mathrm{eV}^{2}$ and $\sin ^{2} 2 \theta_{e \mu}=(0.4-1.5) \times 10^{-2}[8,26]$, we deduce that $M_{2} / M_{1} \lesssim 1$ is required.

The predictions for the $\mu-\tau$ sector follow from Eqs. (3.2) and (3.6). These are given in Table I for central values of the parameters and $m_{\nu_{\mu}}=2 \times 10^{-3} \mathrm{eV}$ (where relevant). The ratio $M_{3} / M_{2}$ is an additional free parameter, although theoretically we expect (see Sec. 4) $1 \lesssim M_{3} / M_{2} \lesssim 100$ : we took $M_{3} / M_{2}=10$ in Table I. The last column gives the cosmic relic density of $\tau$-neutrinos, where $h$ is the Hubble parameter $(0.5 \leq h \leq 1)$. This quantity is simply given by $\Omega_{\nu} h^{2}=m_{\nu} / 91.5 \mathrm{eV}$ [28]. We find it truly amazing that several apparently unrelated experimental measurements and theoretical estimates - solar neutrinos, the MSW mechanism, the see-saw mechanism, and estimates of $m_{t}$ converge to give $\Omega_{\nu} \sim 0.3$ in agreement with the COBE data for $h \sim 0.5$ to 1 . Either this is a remarkable and vicious coincidence, or ....

The current limits [29] on $\nu_{\mu}-\nu_{\tau}$ oscillations exclude values of $\sin ^{2} 2 \theta_{\mu \tau}>4 \times 10^{-3}$ for $\Delta m_{\nu_{\mu}-\nu_{\tau}}^{2} \gtrsim 50 \mathrm{eV}^{2}$, with considerably weaker upper bounds for smaller values of $\Delta m_{\nu_{\mu}-\nu_{\tau}}^{2}$. The proposed CHORUS [16] and NOMAD [17] experiments at CERN and P803 [18] at Fermilab plan to probe values of $\sin ^{2} 2 \theta_{\mu \tau}$ one order of magnitude lower than the current experimental upper bound, with a similar sensitivity to $\Delta m_{\nu_{\mu}-\nu_{\tau}}^{2}$. This means (see Table I) that the predictions for the simple model presented in this section should be fully testable by these new experiments. 
Note that since $m_{\nu_{\tau}} \propto\left(M_{3} / M_{2}\right)^{-1}, M_{3} / M_{2} \sim 1$ would give a $\tau$-neutrino relic density a factor of 10 larger than the values given in Table I and therefore in conflict with current cosmological observations, which require $\Omega_{\nu}<1$ and appear to favor $\Omega_{\nu} \sim 0.3$. Conversely, values of $M_{3} / M_{2} \sim 100$ would make the $\tau$-neutrinos cosmologically uninteresting, even though their mixing with $\mu$-neutrinos would be enhanced by a factor of 10 relative to Table I, but still unconstrained experimentally [29] due to the smallness of $\Delta m_{\nu_{\mu}-\nu_{\tau}}^{2}$ (a factor of 100 smaller than in Table I).

\section{The flipped see-saw mechanism}

We now describe an underlying see-saw mechanism which can produce the above phenomenologically interesting neutrino mass ratios. We do this in the simplest unified supersymmetric extension of the Standard Model which predicts non-zero neutrino masses, namely in the context of flipped $S U(5)[30,19]$. In this model the see-saw matrix $[31,32]$ for each generation involves three fields: $\nu_{i}, \nu_{i}^{c}, \phi_{i}$, where $\phi_{i}$ is an $S U(5) \times U(1)$ singlet field, as follows

$$
\left.\begin{array}{ccc}
\nu_{i} & \nu_{i}^{c} & \phi_{i} \\
\nu_{i}^{c} & \phi_{i} \\
0 & m_{u i} & 0 \\
m_{u i} & \lambda_{9 i} M_{U}^{2} / M_{n r} & \lambda_{6 i} M_{U} \\
0 & \lambda_{6 i} M_{U} & \mu_{i}
\end{array}\right),
$$

where the various entries come from the following flipped $S U(5)$ couplings

$$
\begin{aligned}
& \lambda_{u i} F_{i} \bar{f}_{i} \bar{h} \rightarrow m_{u i} \nu_{i} \nu_{i}^{c}, \\
& \lambda_{6 i} F_{i} \bar{H} \phi_{i} \rightarrow \lambda_{6 i} \bar{V} \nu_{i}^{c} \phi_{i} \approx \lambda_{6 i} M_{U} \nu_{i}^{c} \phi_{i}, \\
& \lambda_{9 i} \frac{1}{M_{n r}} F_{i} F_{i} \bar{H} \bar{H} \rightarrow \lambda_{9 i} \frac{\bar{V}^{2}}{M_{n r}} \nu_{i}^{c} \nu_{i}^{c} \approx \lambda_{9 i} \frac{M_{U}^{2}}{M_{n r}} \nu_{i}^{c} \nu_{i}^{c},
\end{aligned}
$$

and the $\mu_{i} \phi_{i} \phi_{i}$ mass term. In these expressions, $F_{i}, \bar{f}_{i}$ are the usual $\mathbf{1 0}, \overline{\mathbf{5}}$ matter fields, $H, \bar{H}$ are the $\mathbf{1 0}, \overline{\mathbf{1 0}} S U(5) \times U(1)$ breaking Higgs representations whose neutral components $\left(\nu_{H}^{c}, \nu_{\bar{H}}^{c}\right)$ acquire vacuum expectation values $\left(V=\bar{V} \approx M_{U}\right)$, and $M_{n r} \approx 10^{18} \mathrm{GeV}$ is the scale of calculable [33] non-renormalizable terms in the superpotential [34]. The calculable non-renormalizable couplings are a feature of string-derived flipped $S U(5)$ models that we expect on general grounds [33,34] to have counterparts in other string-derived models [35]. The light eigenvalue of the $3 \times 3$ see-saw matrix is simply given by

$$
m_{\nu_{i}} \approx \frac{m_{u i}^{2}}{M_{i}}
$$


with

$$
M_{i}=\lambda_{6 i}^{2} \frac{M_{U}^{2}}{\mu_{i}}\left(1-\frac{\lambda_{9 i}}{\lambda_{6 i}^{2}} \frac{\mu_{i}}{M_{n r}}\right) .
$$

Neglecting the higher-order term (i.e., setting $\left.\lambda_{9 i} \equiv 0\right)$ we find

$$
\frac{M_{3}}{M_{2}}=\frac{\lambda_{63}^{2}}{\lambda_{62}^{2}} \frac{\mu_{2}}{\mu_{3}} .
$$

In string models it is quite common to have a hierarchical set of Yukawa couplings, in that usually only the third generation gets $\mathcal{O}(1)$ couplings; the first and second generation Yukawa couplings are suppressed by powers of $\mu / M_{n r} \sim 1 / 10$ [34]. The masses $\mu_{i}$ may or may not obey any such hierarchy. In specific models we then expect $\lambda_{62} / \lambda_{63} \sim 1 / 10$ and therefore $M_{3} / M_{2} \sim 1-100$ if we also allow for a possible hierarchy in the $\mu_{i}$. We note in passing the key role of the $\lambda_{6}$ couplings (4.2b), which are allowed (even compulsory) in flipped $S U(5)$, but whose phenomenological importance has been hidden until now.

Let us now see if a $\tau$-neutrino mass $\sim 10 \mathrm{eV}$ can be obtained in this model. From Eq. (4.3) we have

$$
m_{\nu_{\tau}}=\frac{m_{t}^{2}\left(M_{U}\right)}{M_{3}}=\frac{m_{t}^{2}}{\eta\left[1-\left(m_{t} / 190\right)^{2}\right]} \frac{1}{M_{3}},
$$

which gives $m_{\nu_{\tau}} \sim 1-10 \mathrm{eV}$ for $M_{3} \sim 10^{12} \mathrm{GeV}$ (with $\eta \approx 10$ ). Also, from Eq. (4.4) $M_{3} \sim \lambda_{63}^{2} M_{U}^{2} / \mu_{3} \sim 10^{12} \mathrm{GeV}$ for $M_{U} \sim 10^{15} \mathrm{GeV}, \mu_{3} \sim 10^{17} \mathrm{GeV}$, and $\lambda_{63} \sim 1 / 3$, all perfectly reasonable numbers.

\section{Summary}

We have emphasized in this note that the MSW [9] interpretation [8] of the GALLEX solar neutrino data [1], extrapolated by a general GUT see-saw mechanism [10,11], is consistent with the suggestion from COBE [14] that there may be a hot component in the Dark Matter [13], namely a $\nu_{\tau}$ weighing $\mathcal{O}(10 \mathrm{eV})$. Furthermore, a plausible texture of neutrino mixing angles, also motivated by GALLEX, suggests that $\nu_{\mu}-\nu_{\tau}$ mixing would be observable in the CHORUS [16] and NOMAD [17] neutrino oscillation experiments now being prepared at CERN. We have also presented a specific realization of this general see-saw mechanism in the context of flipped $S U(5)[30,19]$.

We realize that neither the MSW interpretation of the GALLEX data nor the cocktail interpretation of the COBE data are at all sure, let alone a GUT see-saw mechanism. 
Nevertheless, we find these convergent indications impressive, and hope they encourage our experimental colleagues.

Acknowledgements: This work has been supported in part by DOE grant DE-FG0591-ER-40633. The work of J.L. has been supported in part by an ICSC-World Laboratory Scholarship. The work of D.V.N. has been supported in part by a grant from Conoco Inc. 


\section{References}

[1] GALLEX Collaboration, P. Anselmann et. al., preprint GX 1-1992 (1992).

[2] R. Davis, Jr., et. al., in Proceedings of the 21th International Cosmic Ray Conference, Vol. 12, edited by R. J. Protheroe (University of Adelaide Press, Adelaide, 1990), p. 143.

[3] K. S. Hirata, et. al., Phys. Rev. Lett. 65 (1990) 1297 and Phys. Rev. Lett. 66 (1991) 9.

[4] A. I. Abazov, et. al., Phys. Rev. Lett. 67 (1991) 3332.

[5] J. N. Bahcall and R. N. Ulrich, Rev. Mod. Phys. 60 (1988) 297; S. Turck-Chieze, S. Cahen, M. Casse, and D. Doom, Astrophysical J. 335 (1988) 415.

[6] D. B. Guenther, P. Demarque, Y.-C. Kim and M. H. Pinsonneault, Astrophys. J. 387 (1991) 377, but see D.R.O.Morrison, CERN preprint PPE/92-109 (1992).

[7] See, e.g., S. Bludman, D. Kennedy, and P. Langacker, Nucl. Phys. B 374 (1992) 373 and Phys. Rev. D 45 (1992) 1810.

[8] GALLEX Collaboration, P. Anselmann et. al., preprint GX 2-1992 (1992).

[9] L. Wolfenstein, Phys. Rev. D 17 (1978) 2369 and Phys. Rev. D 20 (1979) 2634; S. P. Mikheyev and A. Yu. Smirnov, Yad. Fiz. 42 (1985) 1441 and Nou. Cim. 9C (1986) 17.

[10] T. Yanagida, Prog. Theo. Phys. B135 (1978) 66.

[11] M. Gell-Mann, P. Ramond, and R. Slansky, in Supergravity, ed. P. van Nieuwenhuizen and D. Freedman (North Holland, Amsterdam 1979), p. 315.

[12] M. Fukugita, M. Tanimoto, and T. Yanagida, Yukawa Institute preprint YITP/K-983 (1992); K.S. Babu and Q. Shafi, Bartol preprint BA-92-27 (April 1992) .

[13] R. Schaefer and Q. Shafi, Bartol preprint BA-92-28 (1992); G. Efstathiou, J. R. Bond, and S. D. M. White, Oxford University preprint OUAST/92/11 (1992); A. N. Taylor and M. Rowan-Robinson, Queen Mary College preprint, (1992); M. Davis, F.J. Summers and D. Schlegel, Berkeley preprint CfPA-TH-92-016 (1992).

[14] G. F. Smoot, et. al., COBE preprint (1992); E. L. Wright, et. al., COBE preprint (1992).

[15] D. V. Nanopoulos and K. Olive, Nature 327 (1987) 487.

[16] CHORUS collaboration, N. Armenise et al., CERN-SPSC/90-42 (1990).

[17] NOMAD collaboration, P. Astier et al., CERN-SPSC/91-21 (1991).

[18] FNAL proposal P803, K. Kodama et al., (1991).

[19] I. Antoniadis, J. Ellis, J. Hagelin, and D. V. Nanopoulos, Phys. Lett. B 194 (1987) 231.

[20] H. Georgi and D. V. Nanopoulos, Phys. Lett. B 82 (1979) 392, Nucl. Phys. B 155 (1979) 52, Nucl. Phys. B 159 (1979) 16. 
[21] S. Dimopoulos, L. Hall, and S. Raby, Phys. Rev. Lett. 68 (1992) 1984 and Phys. Rev. D 45 (1992) 4192.

[22] V. Barger, M. Berger, T. Han, and M. Zralek, Phys. Rev. Lett. 68 (1992) 3394.

[23] D. Kennedy, Fermilab preprint FERMILAB-PUB-92/149-T (1992).

[24] L. Durand and J. L. Lopez, Phys. Rev. D 40 (1989) 207.

[25] H. Fritzsch, Phys. Lett. B 70 (1977) 436 and Phys. Lett. B 73 (1978) 317.

[26] S. Bludman, N. Hata, D. Kennedy, and P. Langacker, University of Pennsylvania preprint UPR-0516T (1992).

[27] J. Ellis, G. L. Fogli, and E. Lisi, Phys. Lett. B 274 (1992) 456.

[28] See e.g., E. Kolb and M. Turner, The Early Universe (Addison-Wesley, 1990).

[29] R. Maschuw, Proc. Joint International Symposium and Europhysics Conference on High Energy Physics, Geneva 1991, eds. S. Hegarty, K. Potter and E. Quercigh (World Scientific, Singapore, 1992), vol. I, p. 619.

[30] S. Barr, Phys. Lett. B 112 (1982) 219, Phys. Rev. D 40 (1989) 2457; J. Derendinger, J. Kim, and D. V. Nanopoulos, Phys. Lett. B 139 (1984) 170.

[31] G. Leontaris, Phys. Lett. B 207 (1988) 447; G. Leontaris and D. V. Nanopoulos, Phys. Lett. B 212 (1988) 327; G. Leontaris and K. Tamvakis, Phys. Lett. B 224 (1989) 319; S. Abel, Phys. Lett. B 234 (1990) 113; I. Antoniadis, J. Rizos, and K. Tamvakis, Phys. Lett. B 279 (1992) 281.

[32] J. L. Lopez and D. V. Nanopoulos, Phys. Lett. B 268 (1991) 359.

[33] S. Kalara, J.L. Lopez and D.V. Nanopoulos, Phys. Lett. B 245 (1990) 421; Nucl. Phys. B 353 (1991) 650.

[34] J. L. Lopez and D. V. Nanopoulos, Phys. Lett. B 251 (1990) 73.

[35] See, e.g., M. Cvetic and P. Langacker, Univ. of Pennsylvania preprint, UPR-505-T (1992). 